\title{
Positive Psychological Experiences in Allogeneic Hematopoietic Stem Cell Transplantation
}

Short Title: Positive Psychological Experiences in HSCT

Hermioni L. Amonoo, MD, MPP ${ }^{1,2,3}$, Lydia A. Brown, $\mathrm{PhD}^{4}$, Carlyn F. Scheu, BS ${ }^{5}$, Rachel A. Millstein, $\mathrm{PhD}^{2,5}$, William F. Pirl, MD, MPH ${ }^{1,2}$, Halyna L. Vitagliano, MD, MSci ${ }^{1,2}$, Joseph H. Antin, MD ${ }^{2,6}$, Jeff C. Huffman, $\mathrm{MD}^{2,5}$

${ }^{1}$ Dana-Farber Cancer Institute, Department of Psychosocial Oncology, Brookline, Massachusetts

${ }^{2}$ Harvard Medical School, Boston, Massachusetts

${ }^{3}$ Brigham and Women's Hospital, Department of Psychiatry, Boston, Massachusetts

${ }^{4}$ University of Melbourne, Melbourne School of Psychological Sciences, Melbourne, Australia

${ }^{5}$ Massachusetts General Hospital, Department of Psychiatry, Boston, Massachusetts

${ }^{6}$ Dana-Farber Cancer Institute, Stem Cell/Bone Marrow Transplantation Program, Division of

Hematologic Malignancy, Brookline, Massachusetts

\author{
Corresponding Author Information: \\ Hermioni Lokko Amonoo, MD, MPP \\ 60 Fenwood Rd, $4^{\text {th }}$ Floor \\ Boston, MA 02115 \\ e-mail: hermioni_lokko@dfci.harvard.edu
}

This is the author manuscript accepted for publication and has undergone full peer review but has not been through the copyediting, typesetting, pagination and proofreading process, which may lead to differences between this version and the Version of Record. Please cite this article as doi: $10.1002 /$ pon.5128

This article is protected by copyright. All rights reserved. 


\begin{abstract}
$\underline{\text { Abstract }}$
Objective: Positive psychological constructs (e.g., optimism, hope) have been associated with superior health outcomes in the recovery from hematopoietic stem cell transplantation (HSCT) in prior work. However, such studies have not examined a broad range of positive psychological constructs, their sources or evolution over time, or their connections with key health behaviors. We used semi-structured qualitative interviews in $25 \mathrm{HSCT}$ patients to explore the nature and sources of positive psychological experiences during the first 100-days after transplantation.

Methods: Participants completed interviews during their HSCT hospitalization and approximately 100 days after transplantation. Transcribed interviews were organized and analyzed using NVivo 12 software by two coders $(\mathrm{K}=.92)$. Interviews focused on the breadth of positive psychological experiences, their sources and evolution during the 100 days, and participants' perceived impact of positive constructs on health behaviors.

Results: Several positive psychological constructs especially gratitude, determination and optimism were frequently expressed. Family support and deliberate participation in pleasant and meaningful activities were the two primary sources of positive psychological experiences after HSCT. At baseline, participants consistently reported gratitude for their donors while follow-up was consistently characterized by hope for cure and return to baseline level of functioning. Additionally, participants related bidirectional relationships between positive psychological well-being experiences and completion of health behaviors over time.
\end{abstract}


Conclusions: As one of the first qualitative studies characterizing the breadth, sources, and evolution of positive psychological experiences in HSCT patients, these findings add to the literature aimed at understanding psychological well-being in this complex and growing patient population.

\section{$\underline{\text { Keywords }}$}

Cancer, Psychosocial Oncology, Hematologic Malignancy, Positive Psychology, Positive Psychological Well-being, Positive Emotional States, Positive Affective States, Hematopoietic Stem Cell Transplant, Positive Psychological Interventions, Positive Constructs 


\section{Background}

Hematopoietic stem cell transplantation (HSCT) provides a potential cure for numerous patients with hematologic malignancies. Approximately 8,000 allogeneic and 14,000 autologous HSCTs are performed in the U.S. each year and the number of transplants is projected to increase significantly. $(1,2)$ Despite the great promise of HSCT, recovery from the transplant (especially allogeneic transplants) is intensive, challenging, and fraught with many complications that impede patients' function and quality of life.(3-5) There are substantial individual differences in coping and quality of life after allogeneic transplantation, with medical (e.g., graft versus host disease, rehospitalization) and psychosocial (e.g., social support) factors contributing to these individual differences.

Psychological factors are very important to HSCT recovery, and the majority of prior studies have examined the impact of negative psychological syndromes.(6) Such studies have found that significant psychological distress accompanies HSCT, and depression, anxiety, or symptoms of posttraumatic stress disorder (PTSD) result in suboptimal health outcomes and increased mortality. $(7,8)$ 
However, not all patients experience clinical psychiatric disorders in the recovery following a stem cell transplant, yet psychiatric factors can still greatly impact their quality of life and recovery. In recent years, there have been increased efforts to explore and understand factors that influence, maintain and enhance the positive psychological well-being (PPWB) of patients recovering from HSCT, given that high levels of emotional well-being and resilience are needed for successful recovery.(9) Beyond mitigating the effects of negative affective states such as depression and anxiety, PPWB constructs (e.g., optimism, positive affect, gratitude, meaning) have been independently associated with better outcomes such as increased quality of life,(10) decreased depression and anxiety,(11) decreased days to neutrophil engraftment(12) and decreased mortality(13) in HSCT patients.

Positive psychology is the science and study of an individual's strengths and values that enable them to thrive and flourish, while positive psychological well-being (PPWB) constructs are cognitive or emotional states or traits which are markers of flourishing or optimal well-being.(14) Different PPWB constructs may have different effects on health and recovery in HSCT patients. Although some prior studies have explored the relationship between a small number of PPWB constructs (such as optimism/hope and positive affect) and health outcomes in this patient population, $(6,15-17)$ in depth qualitative studies that focus on the breadth, evolution, and sources of PPWB experiences in this population are lacking. Furthermore, studies on how PPWB constructs relate to partaking in key health behaviors required for successful recovery are limited. Such work on deeply understanding the nature of PPWB states and traits following HSCT would add to the psychological well-being literature in this population and could ultimately inform the development of impactful psychological interventions that would significantly improve psychological well-being in the HSCT recovery. 
The HSCT process involves several important periods including: a) the transplant hospitalization (which entails the preparation for the transplant, the transplantation itself and the immediate recovery in the hospital), and 2) the post-discharge period up to the 100-day milestone.(18) The psychological needs of patients vary and evolve during these times. Few prior studies have qualitatively examined the positive psychological experiences in the early post-transplant period through the first 100 days of the transplantation.(18) Hence, in this study we used semi-structured qualitative interviews, first during the transplant hospitalization and then at 100 days after transplant among allogeneic HSCT patients, to explore the breadth, sources and evolution of a wide range of PPWB constructs, along with their impact on outcomes during an important transition period in the HSCT recovery.

\section{Methods}

\section{Study Design}

We utilized a qualitative research design to investigate the longitudinal positive emotional experiences of HSCT patients in the first 100 days after their allogeneic transplant. Hour-long, semistructured qualitative interviews were conducted to explore patients' positive emotional experiences at two key time points: 1) towards the end of their HSCT hospitalization (baseline), and 2) at 100 days after transplantation (follow-up). This study followed the consolidated criteria for conducting and reporting qualitative research (COREQ) guidelines,(19) and the study was registered at clinicaltrials.gov (NCT\#02737761). This research was conducted following all guidelines of the Declaration of Helsinki. This study was approved by the institution's Office for Human Research Studies (17-154).

\section{Participant Recruitment}


Participants were adult patients with hematologic malignancies hospitalized for allogeneic stem cell transplantation at an urban academic cancer center between September 2017 and December 2017. All participants provided written informed consent prior to completion of any study procedures. Exclusion criteria included inability to speak English, an active major depressive episode, psychosis or substance use disorder [diagnosed by the Mini International Neuropsychiatric Interview (MINI)(20)], cognitive deficits [assessed with a six-item cognitive screen (21)] impeding ability to provide informed consent or participate adequately in the study, or medical conditions precluding interviews.

\section{Semi-Structured Interviews}

At each time point, participants completed a semi-structured interview that lasted approximately 60 minutes. All baseline interviews were conducted in person prior to patient discharge from the transplant hospitalization (mean of 12 days after their transplant). Most follow-up interviews were conducted via phone. Baseline interviews were completed between September 2017 and February 2018 and all follow-up interviews were completed between December 2017 and May 2018. All interviews (completed by HA) were recorded, transcribed verbatim and uploaded to QSR NVivo 12 software (QSR International, Melbourne, Australia) for analysis.

Semi-structured interview guides were developed based on the research group's clinical experience with HSCT and prior qualitative research investigating emotional experiences in the context of chronic illness.(22, 23) Overall, the interviews examined participants' positive emotional experiences associated with illness and treatment. To contextualize emotional experiences, participants were also invited to share the impact of their illness and treatment on quality of life. Specific topics at both time points included, 1) PPWB in the context of illness and HSCT, 2) sources of PPWB, 3) impact of PPWB 
on daily life, and 4) impact of illness and treatment on various domains of life. Additionally, at follow-up, topics pertaining to differences in quality of life from the transplant hospitalization to outpatient level of care were explored. Participant expressions of emotional states were recorded and coded verbatim without any interpretations by the study team to avoid potential bias. All content relating to emotional experiences was coded and analyzed.

\section{Data Analysis}

We utilized directed content analysis, a descriptive approach to qualitative data analysis.(24) Content analysis is used widely for examining the breadth and scope of patient experiences and themes derived from data that can be extracted with an established framework from pre-existing literature.(25) We used the NVivo software for data organization, coding, and analysis. Two authors [a psychiatrist (HA) and a clinical psychologist (LB)] developed the codebook in consultation with qualitative research experts and the senior author $(\mathrm{JH})$, prior to independently coding all interviews between July 2018 and October 2018. Complete sentences were used as the unit of coding. Codes were informed by both the interview guide and qualitative interview data. The coding structure was modified after reading a small number of transcripts several times to test the codes, and an evidence trail was documented.

Weekly meetings were held during this time to discuss emerging themes from the codes and to adjudicate discrepancies while reviewing interview transcripts to ensure that the themes reflected the original data. In addition to deriving core interview themes, we resolved discrepancies using transcript review, discussion, and consensus. Inter-rater reliability of coding $(\kappa=0.92)$, was calculated after discrepancies were arbitrated. With a sample size of 25 patients at baseline and 21 patients at follow-up, thematic saturation was achieved. 


\section{$\underline{\text { Results }}$}

\section{Demographics}

Thirty-six patients were approached, two were ineligible due to limited English proficiency and severity of illness, nine declined and 25 (71\% of potentially eligible participants) ultimately enrolled, all of whom completed the baseline interviews prior to their discharge from the transplant hospitalization. At follow-up, 21 participants (84\%) completed the interviews. See online supplemental materials for Figure 1 and Table 1 which provide further details about patient enrollment and baseline participant characteristics respectively. Participants had a mean age of 50.4 (SD 14.2), 44\% were women, $60 \%$ were married, and $80 \%$ were White. Acute myeloid leukemia (44\%) was the most common hematologic malignancy, unrelated and matched unrelated donors were the most common transplant sources and most (84\%) participants' GVHD severity was zero.

\section{Positive Psychological Experiences}

Participants reported several positive psychological experiences (including gratitude, optimism, and positive affect) during the first 100 days of their recovery from their transplant. Some of the commonly expressed positive psychological experiences at baseline included hope, gratitude and optimism. Happiness, feeling blessed and fortunate or lucky was consistently reported at follow-up. Gratitude was consistently reported at both time points. See online supplemental materials for Table 2 which provides further details about themes and subthemes of positive psychological experiences reported consistently at both timepoints. 


\section{Hope}

Participants consistently reported hope for their response to treatment. Some participants expressed hope about their treatment and ability to return to premorbid level of functioning. At baseline, such hope was primarily focused on hope for a cure:

"My graft can take on any cancer cells that appear. So I guess you can say I'm hopeful, a little bit hopeful.” (ID 2)

Participants also reported hope, especially at follow-up, that they would return to their pre-transplant level of functioning:

“...that I'm going to get out of the hospital and be better physically. I hope I'll be able to resume some, if not all, of my previous activities." (ID 20)

\section{Gratitude}

Participants expressed gratitude pertaining to their health, clinicians, social support, and faithbased factors. At baseline, several participants expressed gratitude for their stem cell donors:

"I haven't been able to express it to that guy who gave me the bone marrow, but there's gratitude in my heart, just not expressed yet." (ID 10)

Some participants at follow-up were also grateful for the support of family and friends during the entire treatment process:

“...I'm grateful for my friends and family.” (ID 4)

\section{Optimism}

Participants expressed optimism about their treatment and recovery. One participant reported their level of optimism was influenced by the quality of care from both the institution and clinical team: 
“...The medical staff. ... they know how to treat you. They know how to take care of you. ... it instills a sense of confidence, which inherently creates a sense of optimism and positivity too..." (ID 19)

At baseline, more participants reported feeling optimistic about their recovery in the setting of reduced physical symptoms and ability to engage in enjoyable activities:

"Of course. I mean I'm optimistic all the time. It's good when I do get to go and work-out with my buddy and it feels good to go for a drive." (ID 13)

\section{Positive Affect}

Participants shared various experiences with positive affect and attitudes pertaining to multiple aspects of recovery. More participants at follow-up reported positive affect in the setting of good response from their treatment and lack of complications:

“... When I hear good news, that always gives me positive feelings...” (ID 12)

Interestingly, at baseline, more participants recognized that their own positive affect then enriched caregiver and family well-being and facilitated loved ones' ability to adjust to the burden of their illness. For example, one participant reported:

"Because I've had such a positive attitude since the beginning, that trickles down to my wife ... if you express positivity, the people you deal with will kind of feel that in you and be the same back to you, I think.” (ID 5)

\section{Determination and Perseverance}


Determination and perseverance played a role in participants treatment and recovery in different ways. At baseline, several participants articulated how determination and perseverance would impact their recovery as:

“And especially at my age, I knew I could push through the difficult stages to get to transplant. And now the transplant's over. It was certainly all worth it.” (ID 19)

At follow-up, participants emphasized that determination and perseverance helped them to get through the post-transplant care recommendations (such as routine clinic visits, avoidance of crowded places and adherence to a transplant diet):

“...because I want to get better. And I said I'm going to do whatever I need to do to... I want to accomplish my life. Yeah. That's what I really want." (ID 23)

\section{Sources of Positive Experiences}

The two primary themes for sources of positive experiences spontaneously reported by participants were family and deliberate participation in pleasant and meaningful activities. Internal sources of positivity (such as maintaining a positive outlook on life) as well as support from the medical treatment team were also common themes that became increasingly relevant at day 100. Religion and spirituality were also a pertinent source of positive emotional experiences and well-being in a subset of participants. Supplemental Table 3 provides further details on the themes and subthemes for sources of positive experiences.

\section{Family}

Participants reported family who contributed to feelings of love, joy, connectedness, and reassurance as a primary source of positive experiences. One participant summarized this role of family: 
“My glass is always half empty but my family keeps filling it back up.” (ID 1)

Participants explained that their family helped them to feel supported despite changing intrafamily dynamics (such as moving in with family) caused by the stress of cancer and treatment. One participant noted:

"I'm living with my parents at the moment, and they're both incredibly supportive; very loving, very caring.” (ID 4)

\section{Pleasant and/or meaningful activities}

A wide range of pleasant and/or meaningful activities (including reading, meditation, being outdoors, cooking, and helping others) deliberately engaged in by participants were identified as contributing to positive emotional experiences. For example, one participant noted:

“... whether it's a project or whatever, or just look at the window and it's a sunny day, I try to find something that makes me happy every day to focus on." (ID 7)

Some participants reported consciously choosing pleasant activities to self-generate their own happiness:

“To feel more positive or better? I cook [laughter]. These are stress relievers.” (ID 17)

\section{Clinical team}

Clinicians were an increasingly important and primary source of positive states such as hope and encouragement, indicating an elemental role of healthcare practitioners in the emotional well-being of some patients' lives. For example, one participant shared:

“... support from nurses and doctors too. Gave me confidence." (ID 4)

\section{Religious and spiritual activities}

This article is protected by copyright. All rights reserved. 
Finally, a subgroup of participants reported finding substantial strength through religious and spiritual activities such as prayer and reading the Bible/scriptures, belief in God, and via social support from religious communities:

"So many people say I'm on a prayer list at this church. I mean, over 100. And it's a really peaceful feeling when you know that you're not in it alone, and there's so many people pulling for you.” (ID 19)

\section{Relationships between Positive Psychological Constructs and Health Behaviors}

We explored potential relationships between positive psychological experiences and health behaviors (such as self-care and physical activity). A variety of positive psychological constructs (such as determination, positive affect) were linked with the execution of health behaviors. Several participants identified strong links between positive emotional states and willingness to engage in health behaviors:

"Thinking positive can get your body to do the things you need it to do. When I first came home, I could barely walk. And just by sitting up, and standing up for a minute, and then sitting down, and standing up, and sitting down, and then standing up and taking a couple of steps-- I mean, by thinking, 'I can do this,' that's what did it.” (ID 10)

Participants also believed that positive psychological states contributed to increased motivation, enabling them to engage more fully in daily life activities that were good for their health and well-being: "Having those happy feelings motivated me, and I physically feel better and better." (ID 4) Several participants suggested a bidirectional relationship between positive constructs and health behaviors in this population. Specifically, physical activity was reported to increase positive affect and self-regard: 
“The walks, getting fresh air, getting outside, getting up, not being stagnant, being somewhat active no matter what that means on any given day. And getting out is important...the more I can do that, I think, the better off it is for my mindset." (ID 19)

\section{Conclusions}

In this study of 25 allogeneic transplant recipients, we used qualitative interviews to understand and elaborate on the positive psychological experiences at two important periods in the recovery following the transplant. This was, to our knowledge, the first qualitative study to examine positive psychological experiences of allogenic transplant recipients, among the first to explore positive constructs longitudinally via multiple assessments in this population,(6) and to examine explicitly the sources of positive psychological experiences in the context of allogeneic transplantation.(26)

We found that beyond positive psychological traits and states such as optimism and positive affect that have been well-studied in HSCT patients, our sample reported numerous additional positive constructs such as gratitude, determination, perseverance, feeling blessed, and confidence. Many participants also expressed that their illness and treatment allowed them to reflect on their lives and to appreciate aspects of living in ways they had not previously felt. Despite the potential challenges (e.g., risk of GVHD, prolonged quarantine status) of the HSCT recovery, $(9,15)$ these patients still reported significant positive psychological experiences. Indeed, positive experiences such as determination and perseverance helped patients to cope with some of these challenges that can accompany the HSCT recovery.

As with prior work examining changes in negative affective states in HSCT,(16, 17, 27-29) participants reported meaningful variations in positive experiences over the first 100 days after 
transplantation. For example, happiness was reported more consistently at follow-up than at baseline. On the contrary, optimism, while present at both time points, was reported less frequently at follow-up. As observed in our cohort, other studies with cancer patients have shown the likelihood for optimism to fluctuate over the course of the cancer care continuum.(30) Numerous studies have highlighted prospective associations between optimism and improved outcomes (including lower depression and anxiety, increased quality of life, and decreased mortality) in the HSCT population.(6) Hence, the potential decrease in report of an important positive emotional trait, such as optimism, justifies the need to explore interventions that could boost optimism and other positive traits in this population. Additionally, gratitude, a less studied positive construct in medical populations was mentioned most consistently at both time points. Gratitude was commonly expressed toward stem cell donors, treatment teams, and sources of personal social support (e.g., family). This is consistent with prior work in other medical populations, which have found gratitude to be common and focused on similar themes.(31)

Studies specifically examining the sources of positive emotional experiences in HSCT patients are lacking. We found that support from family and deliberate engagement in pleasant or meaningful activities were the two primary sources of positive emotional experiences. Considering the important roles that families play in helping patients navigate various elements of their cancer care,(32) it was not surprising that family was one of the two primary sources of positive emotions. $(33,34)$ Participants indicated that family support induced feelings of love, hope, and gratitude, despite the stress that accompanies managing cancer and recovery following the HSCT. This role of family is important given that support-related variables (e.g., marital status) have been found to be independent predictors of cancer related mortality.(32) The deliberate engagement in pleasant and meaningful activities also yielded a variety of positive emotional experiences. While family support may be less modifiable for any given 
patient, interventions that encourage patients to identify and systematically engage in pleasant and meaningful activities could be especially relevant for HSCT patients. $(6,35)$

Participants identified connections between positive psychological attributes and health behaviors, consistent with studies in other medical populations.(31,36) In our cohort, positive constructs such as positive affect and perseverance (less studied in medical populations) were associated with increased motivation to participate in healthy activities such as walking. Consistent with other studies,(36) participants suggested bi-directional relationships between positive emotional experiences and health behaviors, such that physical activity was likewise associated with an increase in positive states and traits.

Clinical Implications. Our findings highlight the need for an empirically based interventions such as positive psychological intervention (PPI) that promotes and specifically targets PPWB constructs (e.g., optimism, gratitude, positive affect, and determination) in the long-term recovery following HSCT. This study is part of efforts to develop and adapt a PPI for HSCT patients. Unlike other medical populations where PPIs can be initiated during a hospitalization, PPIs for HSCT patients would likely have to start after the 100 days of transplantation when patients have less risk for severe complications and have reduced quarantine requirements. Additionally, further studies on interventions that promote a systematic participation in pleasant and meaningful activities within the context of the HSCT recovery could also provide useful insights on how to enhance PPWB in this population. As part of routine psychological well-being assessments, HSCT clinicians could explore PPWB experiences in their patients and emphasize their importance in the HSCT recovery. Kubzansky et al., provide suggestions of questions (e.g., "Do you ever feel grateful about your health? Tell me about it.") about PPWB that can focus on gratitude, optimism, life satisfaction, social support and positive affect in relation to both health 
and life circumstances.(37) In addition to encouraging patients to actively and deliberately engage in meaningful activities and hobbies that can promote PPWB, HSCT clinicians can also provide patients with information about community resources (e.g., information about affinity/activity groups in local community health centers, churches or volunteer organizations) that can facilitate such activities.

Study Limitations. This study had several limitations. The generalizability of our findings is limited by the lack of diversity in study participants as the majority of the patients were non-Latino White, married, and were recruited from a tertiary academic medical center. Although appropriate for a qualitative study, with a sample size that allowed for thematic saturation, the sample of 25 patients limited our quantitative analyses. To maintain a specific focus on a key population and reduce variability, this study only included patients who received allogenic transplants. Hence, patients who receive different kinds of transplants (e.g., autologous transplants, CAR T-cell therapy) may have different positive psychological experiences than those in our sample. Although study participants were likely more expressive of their psychological mindset, $70 \%$ of the potentially eligible patients enrolled, somewhat reducing concerns about selection bias.

In conclusion, this is one of the first qualitative studies in allogeneic transplant patients that characterizes the breadth, sources and evolution of positive psychological experiences in this population. Although prior work aimed at optimizing psychological well-being in the HSCT population has targeted minimizing negative affective states, research on the relationship of positive psychological states with quality of life and health outcomes in this patient population continues to grow. We found that allogeneic transplant recipients report a wide variety of positive psychological experiences that could potentially positively impact several health outcomes. These findings could help researchers and clinicians develop 
interventions to promote psychological well-being and health outcomes in this growing and vulnerable population.

Funding and Acknowledgement. Time for development and completion of this original research were funded by the Harvard Medical School Dupont-Warren Research Fellowship Award (to HA), Harvard Medical School Livingston Research Award (to HA) and grant 1-17-ICTS-099 from the American Diabetes Association and NIDDK R21DK109313 from the National Institutes of Health (to JH).

Financial Disclosure and Conflict of Interest Statement. All authors of this manuscript confirm no financial disclosures and no conflicts of interest.

This article is protected by copyright. All rights reserved. 
Data Availability Statement. The data that support the findings of this study are available from the corresponding author upon reasonable request.

\section{References}

1. Norkin M, Wingard JR. Recent advances in hematopoietic stem cell transplantation. F1000Res. $2017 ; 6: 870$.

This article is protected by copyright. All rights reserved. 
2. Niederwieser D, Baldomero H, Szer J, Gratwohl M, Aljurf M, Atsuta Y, et al. Hematopoietic stem cell transplantation activity worldwide in 2012 and a SWOT analysis of the Worldwide Network for Blood and Marrow Transplantation Group including the global survey. Bone Marrow Transplant. 2016;51(6):778-85.

3. Bhatia S. Long-term health impacts of hematopoietic stem cell transplantation inform recommendations for follow-up. Expert Rev Hematol. 2011;4(4):437-52; quiz 53-4.

4. Omrani AS, Almaghrabi RS. Complications of hematopoietic stem cell transplantation: Bacterial infections. Hematol Oncol Stem Cell Ther. 2017;10(4):228-32.

5. Chaudhary RK, Dhakal P, Aryal A, Bhatt VR. Central nervous system complications after allogeneic hematopoietic stem cell transplantation. Future Oncol. 2017;13(25):2297-312.

6. Amonoo HL, Barclay ME, El-Jawahri A, Traeger LN, Lee SJ, Huffman JC. Positive psychological constructs and health outcomes in hematopoietic stem cell transplantation patients: a systematic review. Biol Blood Marrow Transplant. 2019;25(1):e5-e16.

7. El-Jawahri A, Traeger L, Greer JA, VanDusen H, Fishman SR, LeBlanc TW, et al. Effect of Inpatient Palliative Care During Hematopoietic Stem-Cell Transplant on Psychological Distress 6 Months After Transplant: Results of a Randomized Clinical Trial. J Clin Oncol. 2017;35(32):3714-21.

8. Costanzo ES, Juckett MB, Coe CL. Biobehavioral influences on recovery following hematopoietic stem cell transplantation. Brain Behav Immun. 2013;30 Suppl:S68-74.

9. Ehrlich KB, Miller GE, Scheide T, Baveja S, Weiland R, Galvin J, et al. Pre-transplant emotional support is associated with longer survival after allogeneic hematopoietic stem cell transplantation. Bone Marrow Transplant. 2016;51(12):1594-8.

This article is protected by copyright. All rights reserved. 
10. Kenzik K, Huang IC, Rizzo JD, Shenkman E, Wingard J. Relationships among symptoms, psychosocial factors, and health-related quality of life in hematopoietic stem cell transplant survivors. Support Care Cancer. 2015;23(3):797-807.

11. Wang ZY, Liu L, Shi M, Wang L. Exploring correlations between positive psychological resources and symptoms of psychological distress among hematological cancer patients: a cross-sectional study. Psychol Health Med. 2016;21(5):571-82.

12. Knight JM, Moynihan JA, Lyness JM, Xia Y, Tu X, Messing S, et al. Peri-transplant psychosocial factors and neutrophil recovery following hematopoietic stem cell transplantation. PLoS One. 2014;9(6):e99778.

13. Lee SJ, Loberiza FR, Rizzo JD, Soiffer RJ, Antin JH, Weeks JC. Optimistic expectations and survival after hematopoietic stem cell transplantation. Biol Blood Marrow Transplant. 2003;9(6):389-96. 14. Fredrickson BL. The role of positive emotions in positive psychology. The broaden-and-build theory of positive emotions. Am Psychol. 2001;56(3):218-26.

15. El-Jawahri A, Pidala J, Khera N, Wood WA, Arora M, Carpenter PA, et al. Impact of psychological distress on quality of life, functional status, and survival in patients with chronic graftversus-host disease. Biol Blood Marrow Transplant. 2018;24:2285-92.

16. Kuba K, Esser P, Mehnert A, Johansen C, Schwinn A, Schirmer L, et al. Depression and anxiety following hematopoietic stem cell transplantation: a prospective population-based study in Germany. Bone Marrow Transplant. 2017;52(12):1651-7.

17. Mosher CE, Redd WH, Rini CM, Burkhalter JE, DuHamel KN. Physical, psychological, and social sequelae following hematopoietic stem cell transplantation: a review of the literature.

Psychooncology. 2009;18(2):113-27.

This article is protected by copyright. All rights reserved. 
18. Bevans MF, Mitchell SA, Marden S. The symptom experience in the first 100 days following allogeneic hematopoietic stem cell transplantation (HSCT). Support Care Cancer. 2008;16(11):1243-54.

19. Tong A, Sainsbury P, Craig J. Consolidated criteria for reporting qualitative research (COREQ): a 32-item checklist for interviews and focus groups. Int J Qual Health Care. 2007;19(6):349-57.

20. Pinninti NR, Madison H, Musser E, Rissmiller D. MINI International Neuropsychiatric Schedule: clinical utility and patient acceptance. Eur Psychiatry. 2003;18(7):361-4.

21. Callahan CM, Unverzagt FW, Hui SL, Perkins AJ, Hendrie HC. Six-item screener to identify cognitive impairment among potential subjects for clinical research. Med Care. 2002;40(9):771-81.

22. Huffman JC, DuBois CM, Mastromauro CA, Moore SV, Suarez L, Park ER. Positive psychological states and health behaviors in acute coronary syndrome patients: a qualitative study. $\mathrm{J}$ Health Psychol. 2016;21(6):1026-36.

23. Huffman JC, Moore SV, DuBois CM, Mastromauro CA, Suarez L, Park ER. An exploratory mixed methods analysis of adherence predictors following acute coronary syndrome. Psychol Health Med. 2015;20(5):541-50.

24. Vaismoradi M, Turunen H, Bondas T. Content analysis and thematic analysis: implications for conducting a qualitative descriptive study. Nurs Health Sci. 2013;15(3):398-405.

25. Elo S, Kyngäs H. The qualitative content analysis process. J Adv Nurs. 2008;62(1):107-15.

26. Applebaum AJ, Bevans M, Son T, Evans K, Hernandez M, Giralt S, et al. A scoping review of caregiver burden during allogeneic HSCT: lessons learned and future directions. Bone Marrow Transplant. 2016;51(11):1416-22.

This article is protected by copyright. All rights reserved. 
27. El-Jawahri AR, Vandusen HB, Traeger LN, Fishbein JN, Keenan T, Gallagher ER, et al. Quality of life and mood predict posttraumatic stress disorder after hematopoietic stem cell transplantation. Cancer. 2016;122(5):806-12.

28. Jim HS, Evans B, Jeong JM, Gonzalez BD, Johnston L, Nelson AM, et al. Sleep disruption in hematopoietic cell transplantation recipients: prevalence, severity, and clinical management. Biol Blood Marrow Transplant. 2014;20(10):1465-84.

29. Anderson KO, Giralt SA, Mendoza TR, Brown JO, Neumann JL, Mobley GM, et al. Symptom burden in patients undergoing autologous stem-cell transplantation. Bone Marrow Transplant. 2007;39(12):759-66.

30. Thieme M, Einenkel J, Zenger M, Hinz A. Optimism, pessimism and self-efficacy in female cancer patients. Jpn J Clin Oncol. 2017;47(9):849-55.

31. Huffman JC, Beale EE, Celano CM, Beach SR, Belcher AM, Moore SV, et al. Effects of Optimism and Gratitude on Physical Activity, Biomarkers, and Readmissions After an Acute Coronary Syndrome: The Gratitude Research in Acute Coronary Events Study. Circ Cardiovasc Qual Outcomes. 2016;9(1):55-63.

32. Aizer AA, Chen MH, McCarthy EP, Mendu ML, Koo S, Wilhite TJ, et al. Marital status and survival in patients with cancer. J Clin Oncol. 2013;31(31):3869-76.

33. Hobbs GS, Landrum MB, Arora NK, Ganz PA, van Ryn M, Weeks JC, et al. The role of families in decisions regarding cancer treatments. Cancer. 2015;121(7):1079-87.

34. Berry LL, Dalwadi SM, Jacobson JO. Supporting the Supporters: What Family Caregivers Need to Care for a Loved One With Cancer. Journal of Oncology Practice. 2017;13(1):35-41. 
35. Huffman JC, Millstein RA, Mastromauro CA, Moore SV, Celano CM, Bedoya CA, et al. A Positive Psychology Intervention for Patients with an Acute Coronary Syndrome: Treatment Development and Proof-of-Concept Trial. J Happiness Stud. 2016;17(5):1985-2006.

36. Huffman JC, DuBois CM, Mastromauro CA, Moore SV, Suarez L, Park ER. Positive psychological states and health behaviors in acute coronary syndrome patients: A qualitative study. $\mathrm{J}$ Health Psychol. 2016;21(6):1026-36.

37. Kubzansky LD, Huffman JC, Boehm JK, Hernandez R, Kim ES, Koga HK, et al. Positive Psychological Well-Being and Cardiovascular Disease: JACC Health Promotion Series. J Am Coll Cardiol. 2018;72(12):1382-96.

Supplemental Figure 1 Legend: Participant Enrollment is summarized in this figure

This article is protected by copyright. All rights reserved. 


\section{University Library}

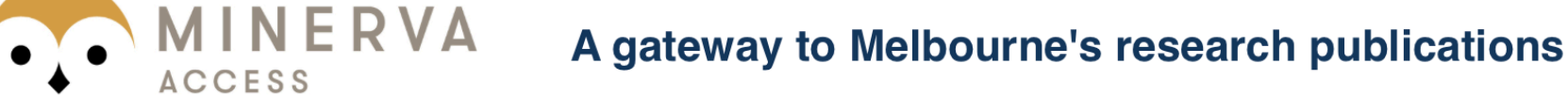

Minerva Access is the Institutional Repository of The University of Melbourne

\section{Author/s:}

Amonoo, HL;Brown, LA;Scheu, CF;Millstein, RA;Pirl, WF;Vitagliano, HL;Antin, JH;Huffman, $\mathrm{JC}$

Title:

Positive psychological experiences in allogeneic hematopoietic stem cell transplantation

Date:

2019-08-01

\section{Citation:}

Amonoo, H. L., Brown, L. A., Scheu, C. F., Millstein, R. A., Pirl, W. F., Vitagliano, H. L., Antin, J. H. \& Huffman, J. C. (2019). Positive psychological experiences in allogeneic hematopoietic stem cell transplantation. PSYCHO-ONCOLOGY, 28 (8), pp.1633-1639. https://doi.org/10.1002/pon.5128.

Persistent Link:

http://hdl.handle.net/11343/285993 